\title{
Structures and diseases
}

\author{
K Ulrich Wendt, Manfred S Weiss, Patrick Cramer \& Dirk W Heinz \\ Structural biology is making significant contributions toward an understanding of molecular constituents \\ and mechanisms underlying human diseases at an atomic resolution, as discussed at the international \\ Murnau Conference on Structural Biology of Disease Mechanisms held in September 2007 in Murnau, Germany.
}

From its very beginning, biostructural research has not only provided tremendous breakthroughs in basic biological processes but also significantly contributed to the understanding of the molecular mechanisms underlying human disease. This was first exemplified when Max Perutz and colleagues elucidated the molecular pathology of human hemoglobin mutations leading to sickle cell anemia ${ }^{1}$. More recently, three-dimensional structures of many human and pathogen proteins have served to guide drug design, with an increasing number of potential target structures determined in the context of structural genomics ${ }^{2}$. Beyond singleprotein approaches, structural biology is now en route toward a high-resolution picture of the cell, assessing protein-protein and protein-nucleic acid complexes at steadily increasing levels of complexity. It is clear that an ever more integrated understanding of the molecular mechanisms of human disease will emerge from this path, although a major challenge lies in translating such knowledge into therapeutic strategies.

In early September 2007, about 180 structural biologists and biochemists

\footnotetext{
K. Ulrich Wendt is in the Department of Chemical and Analytical Sciences at Sanofi-Aventis, D-65926 Frankfurt, Germany. Manfred S. Weiss is at the European Molecular Biology Laboratory, c/o DESY, D-22603

Hamburg, Germany. Patrick Cramer is at the Gene Centre, Ludwig-Maximilians University, D-81377 Munich, Germany. Dirk W. Heinz is in the Division of Structural Biology, Helmholtz Centre for Infection Research, D-38124 Braunschweig, Germany. e-mail:dirk.heinz@helmholtz-hzi.de
}

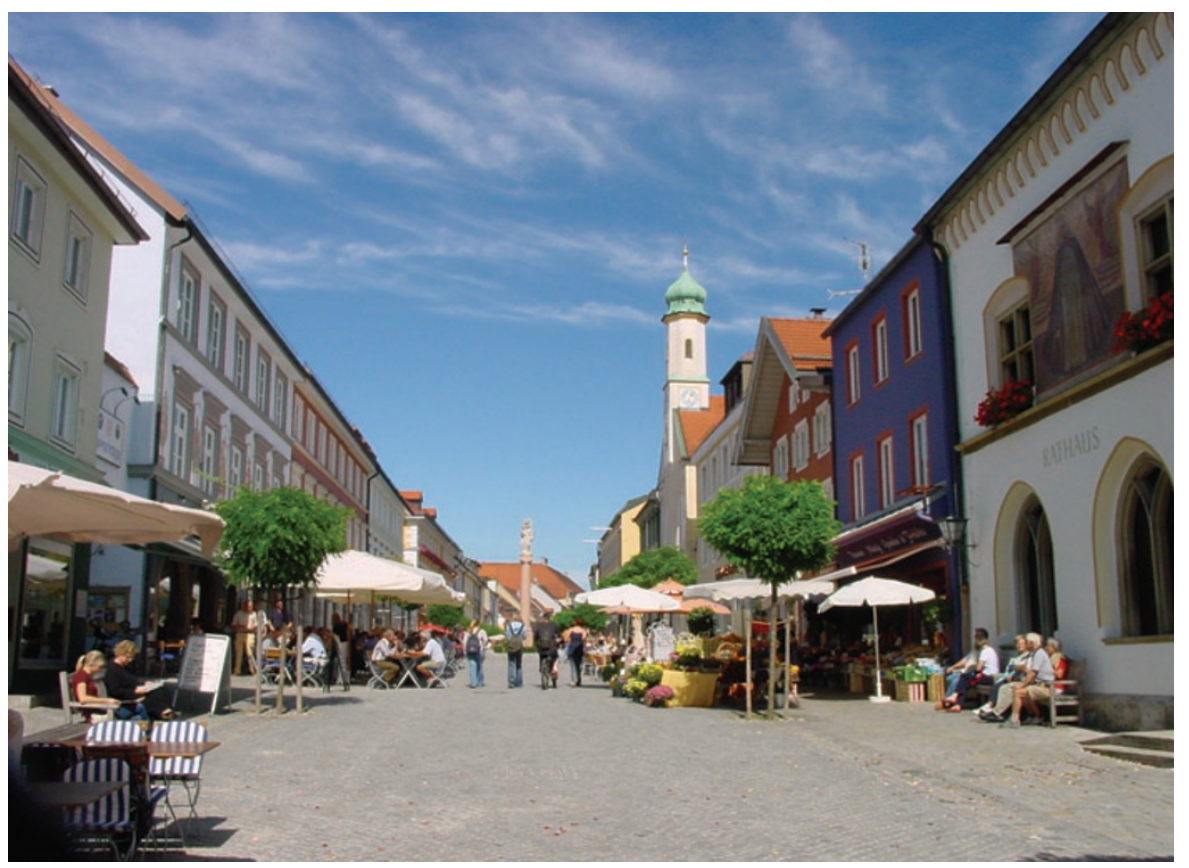

met in the picturesque town of Murnau, located near Staffelsee Lake in the Bavarian alpine upland, to reflect on these questions and discuss recent biostructural data on the molecular determinants of human diseases, including microbial and viral infections, protein misfolding diseases, cancer and metabolic disorders. These topics were addressed during five scientific sessions, two poster sessions and ample time for discussions. The scientific part of the meeting was complemented by a traditional Bavarian-style social program.

The meeting started out with the "Murnau Lecture" held by Wim Hol (University of Washington, Seattle), who gave an impressive overview of the mechanisms of maturation and activity of cholera toxin. With the example of the Medical Structural Genomics of Pathogenic Protozoa (MSGPP) program, he also demonstrated the power of integrating structural biology and biophysical screening approaches to quickly obtain new lead structures for potential drug targets ${ }^{3}$.

The first session, on infectious diseases, was opened by Gabriel Waksman (University College London and Birkbeck College), who investigates the assembly process of Escherichia coli P pili, structures crucial for bacterial virulence. Combining structural and biophysical methods, Waksman and colleagues have shown that the donor strand-exchange mechanism of the P pilus assembly proceeds through a 'zip in-zip out' 
process, involving a transient intermediate complex with a key role in subunit ordering and biogenesis termination ${ }^{4,5}$. This approach also led to the characterization of pilicides, small-molecule inhibitors of pilus formation that interrupt the interactions between the chaperone and the usher ${ }^{6}$, a noncooperative event in the cooperative assembly process. Another surface structure involved in virulence of Gram-negative pathogens such as Shigella flexneri and Yersinia pestis is the type III secretion system (T3SS). Using EM, X-ray crystallography and molecular modeling, Steven Johnson (University of Oxford) presented the first near-atomic model of a T3SS needle ${ }^{7}$ and models of the proteins associated with the needle tip $^{8}$, with the structural basis for the regulation of T3SS assembly under investigation. Erec Stebbins (Rockefeller University) presented a wealth of structural data on the mechanisms of action of T3SS effectors, such as the Yersinia protein kinase A (YpkA), a guanine nucleotide dissociation inhibitor (GDI) for RAC1 GTPase that disrupts the actin cytoskeleton of the host cell ${ }^{9}$. Deshmukh Gopaul (Institute Pasteur) presented data on integron integrases, enzymes that mediate recombination between short symmetric DNA sequences and are thus involved in genetic information exchange between bacteria. The structure of such an enzyme from Vibrio cholerae bound to DNA shows that it recognizes DNA structure rather than a specific sequence ${ }^{10}$. By exploring cell-wall biosynthesis, Gunther Kern and Gautam Sanyal (AstraZeneca) showed that glutamate racemases are suitable targets for narrow-spectrum antimicrobial agents, which are sorely needed against hospitalacquired infections ${ }^{11}$. Hartmut Niemann (Helmholtz Centre for Infection Research and University of Bielefeld) showed how the protein InlB from Listeria monocytogenes exploits the signaling pathways of the receptor tyrosine kinase and protooncogene Met to promote bacterial uptake by the host cell. They find that InlB functionally mimics the natural ligand hepatocyte growth factor/scatter factor (HGF/SF), albeit binding Met at a different domain. The structure also provides insight into the activation mechanism of Met, an important cancer drug target ${ }^{12}$. Stephen Matthews (Imperial College London) presented the latest results on host-cell recognition by the protozoan parasite Toxoplasma gondii, which secretes microneme proteins (MICs) to attach to and penetrate host cells ${ }^{13}$.

Starting the session on viral diseases, Rolf Hilgenfeld (University of Lübeck) reviewed the work from his laboratory on proteases of RNA viruses, such as severe acute respiratory syndrome (SARS) coronavirus and coxsackievirus B3, and also highlighted recent structural data on falcipain-2 from Plasmodium falciparum, discussing implications for the design of active-site directed and allosteric inhibitors for these cysteine proteases ${ }^{14}$. Young Do Kwon from Peter Kwong's group (US National Institutes of Health) shifted the focus to HIV-1, whose ability to evade the human immune system is a major obstacle for vaccine development. The binding site for human receptor CD4 on viral gp120 is accessible, but most antibodies directed to this site are not neutralizing. Kwon compared the structure of gp120 in complex with non-neutralizing antibody $\mathrm{F} 105$ and with neutralizing antibody b12 (ref. 15; Fig. 1), revealing that, upon CD4 binding, a hydrophobic surface in gp120 becomes exposed, to which the non-neutralizing antibody F105 binds. Dennis Bamford (University of Helsinki) explored the architectural principles of capsids from viruses that infect various hosts from bacteria to humans to suggest that early cells were infected with many different viruses and only a limited number of folds have been selected to assemble viable virus coats $^{16}$. Eloise Mastrangelo from Martino Bolognesi's group (University of Milan) presented work on the NS3 proteasehelicase from Kunjin virus, a flavivirus affecting livestock and man. Small-angle X-ray scattering (SAXS) data reveal that domain rearrangements upon RNA binding may explain the unwinding efficiency of NS3 (ref.17). Winfried Weissenhorn (European Molecular Biology Laboratory Grenoble) presented the three-dimensional structure of the rabies virus nucleoproteinRNA complex at $3.5-\AA$ resolution, revealing how these viruses compact and protect their genomes ${ }^{18}$.

Various pathogens are efficiently cleared from the bloodstream by the complement system, an important part of innate immunity. The complement protein $\mathrm{C} 3$ in its activated form (C3b), the subject of recent hot debate among the protein crystallographic community, binds to pathogens and tags them for phagocytosis via the complement receptors (CRIg). Christian Wiesmann (Genentech) presented the structure of the C3b-CRIg complex, revealing the dramatic structural rearrangements that take place during complement activation ${ }^{19}$. Wiesmann and colleagues further demonstrated that CRIg inhibits alternative pathway convertases, a finding with implications for the development of therapeutics targeting the complement system, which is involved in various human diseases.

The session on protein misfolding was started out by Roland Riek (Salk Institute), who recently returned to the Swiss Federal Institute of Technology (ETH, Zurich). Combining solid-state NMR, EM and hydrogen-deuterium exchange methods, Riek has identified fibrillogenic sequences in various proteins, including the amyloid peptide $A \beta_{1-42}$ implicated in Alzheimer's disease $^{20}$. Studies with $\mathrm{A} \beta_{1-42}$ mutants

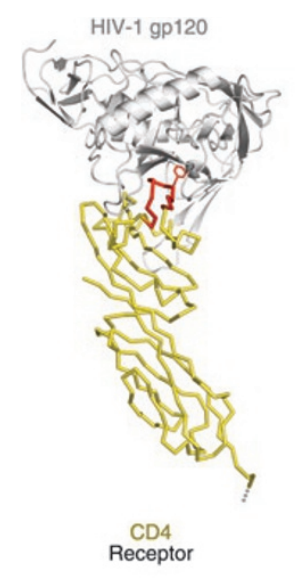

Figure 1 The site on HIV-1 gp120 (gray) of interaction with the CD4 receptor (yellow) represents a conserved accessible surface on HIV-1, and many commonly elicited antibodies compete with CD4 for binding to gp120. However, most of these are weakly neutralizing and relatively impotent against primary HIV-1 isolates. One exception is the b12 antibody: whereas all of the CD4 binding site ligands seem to have an extended loop tipped by a hydrophobic residue (red), b12 recognizes gp120 in a slightly different way than CD4 does. These structures might help design an immunogen that is able to elicit more b12-like antibodies. Figure courtesy of Peter Kwon, US National Institutes of Health. 
indicate that the toxicity of $A \beta_{1-42}$ relates to the morphology of the aggregate formed. Riek also discussed the use of amyloid fibrils in nanotechnology as a deposit form for the slow release of bioactive peptides. Marcus Fändrich (Leibniz Institute, Jena) reported the three-dimensional EM reconstruction of an $A \beta_{1-40}$ amyloid fibril in collaboration with Niko Grigorieff (Brandeis University). At $26-\AA$ resolution, the presented EM map deviates significantly from previous models, with an entirely different fibril cross section. On the basis of fibril classification results, Fändrich also demonstrated that $A \beta_{1-40}$ fibrils can be vastly heterogeneous, which is important when considering the biological or structural properties of a given amyloid sample. Luigi Vitagliano (L' Istituto di Biostrutture e Bioimmagini and Consiglio Nazionale delle Ricerche, Naples) presented insights on fibril models from molecular dynamics calculations, starting from the crystal structure of hexa- or heptapeptides from Eisenberg's laboratory (see below) and showing that the minimally stable oligomer was a pentamer of hexapeptides, with one peptide significantly shielded by the other four ${ }^{21}$. Christian Betzel (University of Hamburg) presented work on aggregated prion proteins, using a cellfree assay that converts cellular PrP into an isoform similar to its infectious form, PrPSc, by mimicking oxidative stress, with SAXS analysis providing structural insights into the mechanism of oligomerization ${ }^{22}$. The session was concluded by a superb presentation by David Eisenberg (University of California Los Angeles), who gave a brief overview of early fibril models derived from cross- $\beta$-diffraction images before moving on to recent work on amyloidogenic peptides. On the basis of about 30 crystal structures of amyloid peptides determined in the Eisenberg laboratory ${ }^{23}$, computational approaches were developed to predict the amyloid propensity of peptide stretches in proteins, identifying LVEALYL from human insulin as a potentially fibrillogenic sequence.

In summary, this session gave a good overview of the recent achievements in this field. Although it might be a long way until a drug against the disease discovered by Aloys Alzheimer is developed and available to patients, it is truly remarkable how much has been learned about a process that a few years ago was the subject of wild speculations.

Alan Fersht (University of Cambridge) opened the session on cancer, reporting on the tumor-suppressor protein p53, which is inactive in about half of all human cancers, in many cases because of mutations that lower the thermal stability of the core domain to below body temperature. Fersht's group has designed compounds to rescue the function of such p53 mutants as a potential cancer therapeutic strategy ${ }^{24}$, with the crystal structures of oncogenic p53 mutants revealing cavities that could be appropriate targets for drugs designed to chemically rescue 553 function. Finally, the combination of SAXS, EM and NMR studies, together with previous crystallographic data, resulted in the complete architecture of the p53 tetramer, which includes large intrinsically unstructured regions ${ }^{25}$. Holger Rehmann (University Medical Centre Utrecht) reported on the structural basis of the regulation of the guanine nucleotide exchange factor Epac by cAMP $^{26}$. Alfred Wittinghofer (Max-Planck Institute, Dortmund) has applied the detailed understanding of the GTPase reaction of Ras to devise potential strategies for cancer therapy. Wittinghofer's group found a small molecule of undisclosed structure that could induce activity of an oncogenic inactive Ras variant in vitro ${ }^{27}$. Guillermo Montoya (Spanish National Cancer Center, Madrid) reported the molecular basis of substrate recognition by polo-like kinase 1 (Plk1) and its implications for centrosomal localization ${ }^{28}$. Claus Kuhn (Gene Center, Munich) reported on the functional architecture of yeast RNA polymerase I, which carries out the synthesis of ribosomal RNA, a question that was unraveled by combining cryo-EM, X-ray crystallography and homology modeling. Biochemical and genetic experiments nicely complemented the structural data, showing how RNA polymerase I deviates in structure and function from RNA polymerase $\mathrm{II}^{29}$.

Finally, Titia Sixma (Netherlands Cancer Institute, Amsterdam), out of a collaboration with Andrea Pichler (Medical University Vienna) and Frauke Melchior (University of Göttingen), reported the crystal structure of a SUMOylated E2, which showed that SUMOylation affects the E2 enzymatic activity by modulating E2's interaction with E1 and that recognition of the SUMOylation site depends on the surrounding structure of the site. Finally, she showed that SUMO can bind to different sites on Ubc9, depending on whether a covalent thioester-linked complex, a modified lysine or a non-covalent complex is formed ${ }^{30}$.

The final session started with structures of extracellular receptors involved in metabolic control and cardiovascular disease. Michael Lawrence (Walter and Eliza Hall Institute) presented the 3.8 - $\AA$ crystal structure of the insulin receptor ectodomain in complex with four Fabs and an insulin mimetic peptide $^{31}$, thereby providing the first view of the spatial arrangement of low- and high-affinity insulin binding sites. Günter Fritz (University of Konstanz) presented the unpublished structure of the ligand binding domain of RAGE, a multiligand receptor for advanced glycation end products, $\mathrm{S} 100$ proteins, HMGB1 and amyloid- $\beta$, whose activation is key to numerous chronic diseases such as diabetes, inflammation, arteriosclerosis and neurodegeneration, making it a potential therapeutic target ${ }^{32,33}$. Armin Ruf (Hoffmann-La Roche) provided a view on the structure-guided design of two newly identified classes of PPAR $\alpha / \gamma$ dual agonists whose profile seems well suited for addressing both hyperglycemia as well as the enhanced cardiovascular risk of diabetic patients ${ }^{34}$.

Annalisa Pastore (Medical Research Council, London) shifted the focus of the session to rare diseases, with her recent findings on the anomalous expansion of polyglutamine motifs as a basis for neurodegenerative misfolding diseases ${ }^{35}$. Antti Haapalainen (University of Oulu) presented structural and functional studies of the human mitochondrial acetoacteyl-CoA thiolase T2, whose loss-of-function mutations result in severe ketoacidosis ${ }^{36}$. Markus Wahl (Max-Planck Institute, Göttingen) combined structural and functional studies in search of a molecular basis for retinitis pigmentosa (RP). The data support a model in which PRP8, a spliceosomal factor whose mutations cause the severe RP13 form, serves as a scaffold for the assembly of other factors such as the DEAD box protein BRR2 and GTPase SNU114, and show that viable RP13-related mutations weaken but do not abolish these interactions ${ }^{37}$. Ryota Kuroki (Japan Atomic Energy Agency) presented recent structural studies on the complex of human granulocyte colony-stimulating factor (GCSF), a cytokine used for treatment of granulopenia, with its receptor. Although various stochiometries had been proposed for this assembly, Kuroki's work underscores the relevance of the 2:2 complex, a result that is in line with thermodynamic and mutational analyses ${ }^{38}$.

Roger Williams (Medical Research Council, Cambridge) closed the session with a comprehensive overview of recent structural results on the cellular ESCRT machinery ${ }^{39}$, which mediates the trafficking of monoubiquitinated proteins to lysosomes via the multivesicular budding (MVB) pathway. This process has a role in the downregulation of cell-surface receptors and the budding 
of HIV and other retroviruses. Williams discussed the molecular understanding reached for many components of the MVB pathway, their molecular assemblies and the sorting signal ubiquitin. Major challenges remain in understanding the higher-level molecular organization of the ESCRT lattice together with a molecular mechanism for vesicle budding. Solution of these challenges will require the application of hybrid methods to close the resolution gap between X-ray structures and current EM reconstructions.

In summary, the 2007 Murnau conference featured 31 presentations and 66 posters on many molecular aspects underlying human disease. Several of the presentations demonstrate how an advanced molecular understanding of disease-relevant factors can open new strategies for both the design of interfering small molecules and screening procedures for such compounds. In other cases, the complexity of supramolecular assemblies solved by structural methods seems to overwhelm our current ability to translate the emerging higher-order molecular view into concrete options for therapeutic intervention. As demonstrated by Hol, Waksman, Fersht, Wittinghofer, Riek and many others, this step can come within reach only when structural biology research is tightly integrated with biophysical, biochemical and cellular studies. This strategy of integrating functional and structural studies of ever more complex cellular processes in search of new therapeutic entry points was reflected in many presentations and discussions at Murnau. For the upcoming meeting in 2010, it may be of interest to focus on the interface of structural and chemical biology, where structural information is used to reveal the action mechanism of new bioactive compounds for improved intervention strategies ${ }^{40}$.

1. Morimoto, H., Lehmann, H. \& Perutz, M.F. Nature 232, 408-413 (1971).

2. Kuhn, P., Wilson, K., Patch, M.G. \& Stevens, R.C. Curr. Opin. Chem. Biol. 6, 704-710 (2002).

3. Bosch, J. et al. J. Med. Chem. 49, 5939-5946 (2006).

4. Remaut, H. et al. Mol. Cell 22, 831-842 (2006).

5. Verger, D. et al. EMBO Rep. 7, 1228-1232 (2006).

6. Pinkner, J.S. et al. Proc. Natl. Acad. Sci. USA 103 , 17897-17902 (2006). (Iv)(Iv)

7. Deane, J.E. et al. Proc. Natl. Acad. Sci. USA 103, 12529-12533 (2006).

8. Johnson, S. et al. J. Biol. Chem. 282, 4035-4044 (2007).

9. Prehna, G., Ivanov, M.I., Bliska, J.B. \& Stebbins, C.E. Cell 126, 869-880 (2006).

10. MacDonald, D., Demarre, G., Bouvier, M., Mazel, D. \& Gopaul, D.N. Nature 440, 1157-1162 (2006).

11. Lundqvist, T. et al. Nature 447, 817-822 (2007).

12. Niemann, H.H. et al. Cell 130, 235-246 (2007).

13. Blumenschein, T.M.A. et al. EMBO J. 26, 2808-2820 (2007).

14. Hogg, T. et al. J. Biol. Chem. 281, 25425-25437 (2006).

15. Zhou, T. et al. Nature 445, 732-737 (2007).

16. Bamford, D.H., Grimes, J.M. \& Stuart, D.I. Curr. Opin Struct. Biol. 15, 655-663 (2005).

17. Mastrangelo, E. et al. J. Mol. Biol. 372, 444-455 (2007).
18. Albertini, A.V. et al. Science 313, 360-363 (2006). 19. Wiesmann, C. et al. Nature 444, 217-220 (2006).

20. Lührs, T. et al. Proc. Natl. Acad. Sci. USA 102 17342-17347 (2005).

21. Esposito, L., Pedone, C. \& Vitagliano, L. Proc. Natl. Acad. Sci. USA 103, 11533-11538 (2006).

22. Redecke. L. et al. Structural characterization of $\beta$-sheeted oligomers formed on the pathway of oxidative prion protein aggregation in vitro. J. Struct. Biol. 157, 308-320 (2007).

23. Sawaya, M.R. et al. Nature 447, 453-457 (2007)

24. Joerger, A.C. \& Fersht, A.R. Oncogene 26, 2226-2242 (2007).

25. Tidow, H. et al. Proc. Natl. Acad. Sci. USA 104 12324-12329 (2007).

26. Rehmann, H., Das, J., Knipscheer, P., Wittinghofer, A. \& Bos, J.L. Nature 439, 625-628 (2006).

27. Bos, J.L., Rehmann, H. \& Wittinghofer, A. Cell 129 865-877 (2007)

28. Garcia-Alvarez, B., de Carcer, G., Ibanez, S., Bragado-Nilsson, E. \& Montoya, G. Proc. Natl. Acad. Sci. USA 104, 3107-3112 (2007).

29. Kuhn, C. et al. Cell 131, 1260-1272 (2007).

30. Knipscheer, P., van Dijk, W.J., Olsen, J.V., Mann, M. \& Sixma, T.K. EMBO J. 26, 2797-2807 (2007).

31. McKern, N.M. et al. Nature 443, 218-221 (2006).

32. Bierhaus, A., Stern, D.M. \& Nawroth, R. Curr. Opin. Investig. Drugs 11, 985-991 (2006).

33. Ostendorp, T. et al. EMBO J. 26, 3868-3878 (2007).

34. Kuhn, B. et al. Bioorg. Med. Chem. Lett. 16 4016-4020 (2006).

35. Nicastro, G., Menon, R., Masino, L., McDonalds, N.O. \& Pastore, A. Proc. Natl. Acad. Sci. USA 102 10493-10498 (2005).

36. Haapalainen, A.M.et al. Biochemistry 46, 4305-4321 (2007).

37. Pena, V., Liu, S., Bujnicki, J., Lührmann, R. \& Wahl, M.C Mol. Cell 25, 615-624 (2007).

38. Tamada, T. et al. Proc. Natl. Acad. Sci. USA 103 , 3135-3140 (2006).

39. Obita, T. et al. Nature 449, 735-739 (2007).

40. Schreiber, S.L. Nat. Chem. Biol. 1, 64-66 (2005). 